\title{
Hypothyroidism conversion to hyperthyroidism: it's never too late
}

\author{
Ehtasham Ahmad1,*, Kashif Hafeez², Muhammad Fahad Arshad3,*, Jimboy Isuga4 and Apostolos Vrettos ${ }^{5}$ \\ 'Endocrinology and Diabetes, Queen Elizabeth the Queen Mother Hospital, Margate, UK, 2Endocrinology and \\ Diabetes, Tunbridge Wells Hospital, Tunbridge Wells, UK, ${ }^{3}$ Endocrinology and Diabetes, Sheffield Teaching Hospitals, \\ Sheffield, UK, ${ }^{4}$ Tunbridge Wells Hospital, Tunbridge Wells, UK, and ${ }^{5}$ St George's University of London Medical \\ School, London, UK \\ *(E Ahmad and M F Arshad contributed equally and are joint first authors) \\ Correspondence \\ should be addressed \\ to E Ahmad \\ Email \\ ehtasham.ahmad@nhs.net
}

\section{Summary}

Primary hypothyroidism is a common endocrine condition, most commonly caused by autoimmune thyroiditis (Hashimoto's disease) while Graves' disease is the most common cause of hyperthyroidism. Hypothyroidism is usually a permanent condition in most patients requiring lifelong levothyroxine treatment. Transformation from Hashimoto's disease to Graves' disease is considered rare but recently been increasingly recognised. We describe a case of a 61-yearold lady who was diagnosed with hypothyroidism approximately three decades ago and treated with levothyroxine replacement therapy. Approximately 27 years after the initial diagnosis of hypothyroidism, she started to become biochemically and clinically hyperthyroid. This was initially managed with gradual reduction in the dose of levothyroxine, followed by complete cessation of the medication, but she remained hyperthyroid, ultimately requiring anti-thyroid treatment with Carbimazole. This case highlights that there should be a high index of suspicion for a possible conversion of hypothyroidism to hyperthyroidism, even many years after the initial diagnosis of hypothyroidism. To our knowledge, this case illustrates the longest reported time interval between the diagnosis of hypothyroidism until the conversion to hyperthyroidism.

\section{Learning points:}

- Occurrence of Graves' disease after primary hypothyroidism is uncommon but possible.

- In this case, there was a time-lapse of almost 28 years and therefore this entity may not be as rare as previously thought.

- Diagnosis requires careful clinical and biochemical assessment. Otherwise, the case can be easily confused for overreplacement of levothyroxine.

- We suggest measuring both anti-thyroid peroxidase (TPO) antibodies and TSH receptor antibodies (TRAB) in suspected cases.

- The underlying aetiology for the conversion is not exactly known but probably involves autoimmune switch by an external stimulus in genetically susceptible individuals.

\section{Background}

Hashimoto's thyroiditis and Graves' disease are the most common autoimmune thyroid conditions and are more common in women than in men (1). Both conditions are characterised by symptoms and signs on the opposite spectrum of the scale and have entirely different appearances on radioactive iodine nuclear imaging studies. Although cases of conversion from hyperthyroidism to hypothyroidism are often 
encountered in clinical practice, it is rare to see conversion of primary hypothyroidism due to Hashimoto's disease to hyperthyroidism due to Graves' disease $(2,3)$. The exact incidence of this conversion is not known due to its presumed rarity, and we could only find a limited number of similar cases in the literature, where this process took place over a variable time course spanning from months to years. Despite its rarity, it is possible that patients developing this conversion could be encountered by primary care physicians as well as hospital-based doctors and may be mistaken for over-replacement of levothyroxine. Treatment is straightforward with anti-thyroid medications once the diagnosis is confirmed.

\section{Case presentation}

We describe the case of a 61-year-old lady diagnosed with primary hypothyroidism back in 1988, based on tiredness, fatigue and a high thyroid-stimulating hormone (TSH) of $7.1 \mathrm{mU} / \mathrm{L}$ (normal: $0.27-4.2 \mathrm{mU} / \mathrm{L}$ ). No concomitant free $\mathrm{T} 4$ or antibodies were checked, and an ultrasound scan was not done at that time. She was managed with levothyroxine replacement therapy $100 \mu \mathrm{g}(1.3 \mu \mathrm{g} / \mathrm{kg} / \mathrm{day})$ since the diagnosis was made. Her comorbidities are type 2 diabetes, hypertension, dyslipidaemia and gout. Her other regular medications include insulin, irbesartan, hydrochlorothiazide, bisoprolol, simvastatin and allopurinol. She works as a social worker in a residential home. She is an ex-smoker, having quit smoking at the age of 24 years and drinks alcohol rarely (less than 14 units/week).

In 2015, and after having been on her usual levothyroxine $100 \mu \mathrm{g}$ daily since the initial diagnosis of hypothyroidism in 1988, her general practitioner (GP) noticed a hyperthyroid picture on routine blood test for the first time after 27 years. That biochemical derangement was attributed to possible over-replacement of levothyroxine. It was decided to gradually reduce the levothyroxine dose, while having periodic monitoring of the thyroid function. At the start of January 2017, it was found that her TSH was completely suppressed (Fig. 1) and that the free T4 rose up to $71 \mathrm{pmol} / \mathrm{L}$ (normal $12-22 \mathrm{pmol} / \mathrm{L}$ ) despite reduction in levothyroxine dose (Fig. 2). The GP completely stopped her thyroid hormone replacement and referred her for specialist's opinion.

This lady was subsequently reviewed in the outpatient endocrinology clinic. On further enquiring, she reported symptoms of excessive tiredness and sweating for last 24 months. She also noticed weight loss

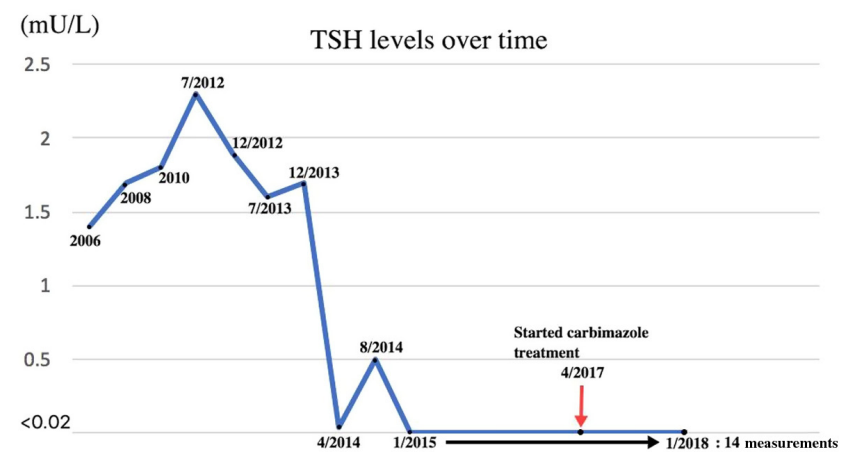

Figure 1

TSH levels over time.

of approximately $10 \mathrm{~kg}$ during the same time. There was no history of palpitations or diarrhoea. Family history was significant for thyroid disease in her father and paternal grandmother. Although she was not aware of the exact details of the nature of the thyroid disease, she reported that her father had an underactive thyroid, which eventually became overactive after unspecified time. On examination, she had fine tremors of her outstretched hands, and there was bilateral exophthalmos with lid lag present. Although she was not complaining of any eye symptoms, exophthalmos was noticed both by primary care physician and the endocrinology consultant. She had a Clinical Activity Score of 2 (mild redness of the conjunctiva and swelling of eyelids). Lid retraction was less than $2 \mathrm{~mm}$. There was no documentation of the degree of exophthalmos. There was diffuse thyromegaly with right lobe more prominent than the left lobe and an audible thyroid bruit. Unfortunately, there was no documented record of the thyroid volume in her retrospectively reviewed clinical notes. It was noticed in

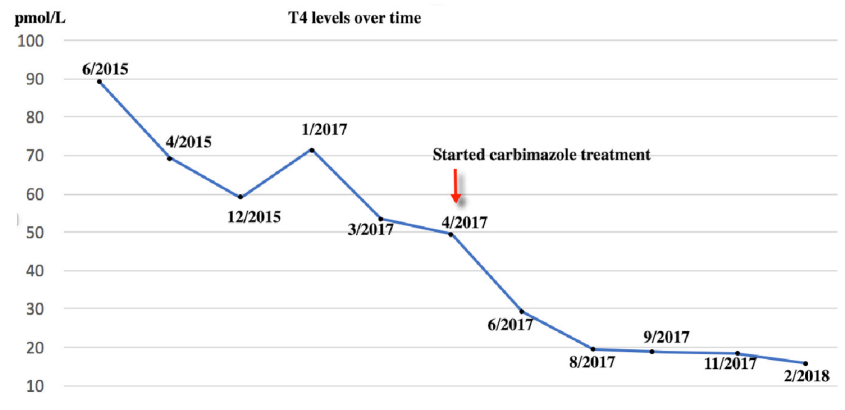

Figure 2

T4 levels over time. She was on a stable dose of levothyroxine of $100 \mu \mathrm{g}$ since diagnosis, until 2016. In 2016, the GP gradually reduced the dose to $75 \mu \mathrm{g}$ daily, and then 75 and $50 \mu \mathrm{g}$ on alternate days. As she remained hyperthyroid, in January 2017, the GP completely stopped the levothyroxine, as the TSH was suppressed and the T4 was $71.5 \mathrm{pmol} / \mathrm{L}$. 
her previous blood results from 2006 onwards that her TSH levels had always been within the normal range on a stable dose of levothyroxine $100 \mu$ g daily until 2015, where they became persistently low (Fig. 1).

Before the onset of the hyperthyroidism symptoms, she had an upper respiratory tract infection, for which she empirically received antibiotics in the community by her GP. No specific investigations like blood or sputum cultures or X-rays were done. However, according to the patient, following this infection, her diabetes became uncontrolled requiring her to go on insulin, her white cell count dropped and then a few weeks later, her thyroid function tests (TFTs) started to become deranged. Beginning from that episode of illness in January 2015, her thyroid biochemistry became inconsistent, gradually shifting to a hyperthyroid picture. A TSH check in August 2014 was $0.5 \mathrm{mU} / \mathrm{L}$. In January 2015, it was $<0.02 \mathrm{mU} / \mathrm{L}$ and on a repeat TFTs in June 2015 the TSH was $<0.02 \mathrm{mU} / \mathrm{L}$ and free T4 was $89.2 \mathrm{pmol} / \mathrm{L}$. This is when she also started developing hyperthyroid symptoms. It is difficult to ascertain what exactly caused this conversion, which happened to coincide with the upper respiratory tract infection, and it is difficult to prove a causal association between those two. However, it is plausible that the infection, probably a viral one, could have switched the conversion from the TSH-blocking antibodies to TSH-stimulating antibodies, making her clinically and biochemically hyperthyroid. Interestingly, her father had underactive thyroid which eventually became overactive. This points to the fact that there may be genetic susceptibility in addition to an environmental trigger causing the conversion from hypothyroidism to hyperthyroidism. Further research is, however, warranted to prove this.

\section{Investigation}

Her blood results have been consistent with hypothyroidism until 2015, when she developed a hyperthyroid picture (Figs 1 and 2). The anti-TPO levels came back as equivocal at $83 \mathrm{IU} / \mathrm{mL}$ (negative $<60$, equivocal: $60-100 \mathrm{IU} / \mathrm{mL}$ ) and the TRAB was elevated at $15.8 \mathrm{IU} / \mathrm{L}(<0.9 \mathrm{IU} / \mathrm{L})$, confirming the autoimmune basis of the underlying problem. No thyroid-stimulating antibody or thyroid-blocking antibody was checked. Only diagnostic, and not serial, TRAB was performed as per local policy. A thyroid uptake scan was suggestive of early Graves' disease (Fig. 3). A full autoimmune screen was not performed but coeliac screen was negative.

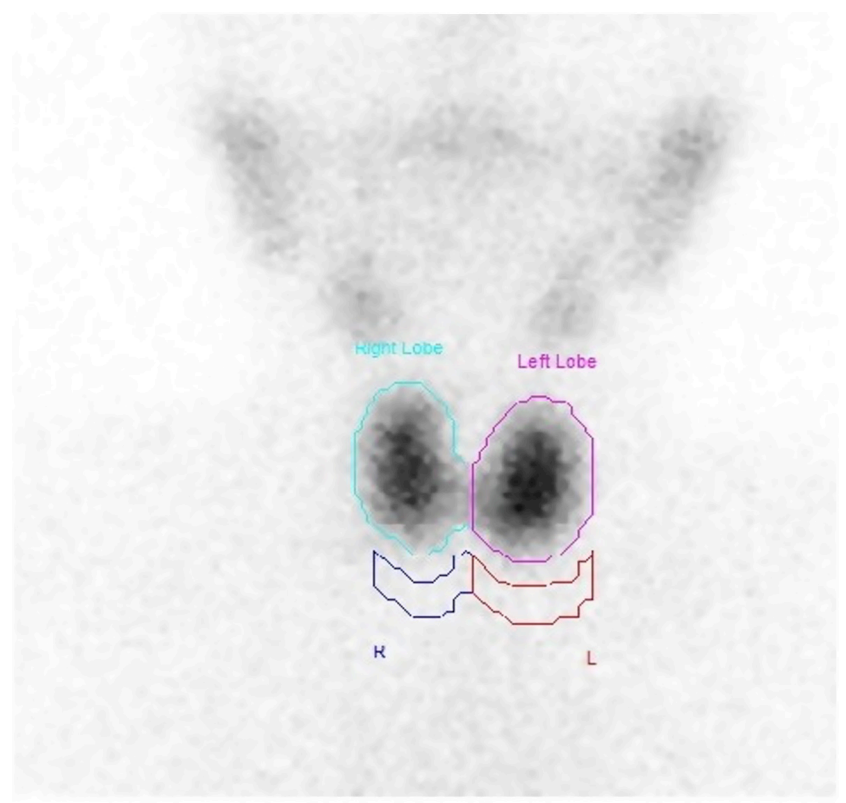

Thyroid uptake is $2 \%$

Right lobe uptake is $1 \%$

Left lobe uptake is $1.1 \%$

Figure 3

Results of thyroid nuclear scan using Technetium 99m. Diffuse homogeneous uptake throughout both lobes of the thyroid with no evidence of toxic nodule or thyroiditis. These appearances could be suggestive of early Graves' disease.

\section{Treatment}

Based on the above results, there was little doubt that patient had underlying Graves' disease now and was commenced on Carbimazole $30 \mathrm{mg}$ once a day in 2017.

\section{Outcome and follow-up}

She was followed up in the endocrine clinic reporting improvement in her symptoms and a weight gain of $6 \mathrm{~kg}$ following the initiation of anti-thyroid treatment, over a period of 4 months. She is now clinically euthyroid. Because of the biochemical and clinical improvement, the dose of Carbimazole was reduced from $30 \mathrm{mg}$ to $20 \mathrm{mg}$ daily. Her latest TFTs on Carbimazole $20 \mathrm{mg}$ daily showed that her TSH has remained suppressed with a free T4 of $15.7 \mathrm{pmol} / \mathrm{L}$ (Fig. 2 and Table 1).

\section{Discussion}

In this case report, we describe a middle-aged lady who was initially diagnosed with hypothyroidism approximately 30 years ago. She remained euthyroid on stable doses of levothyroxine before converting to hyperthyroidism 
Table 1 TFTs since starting Carbimazole.

\begin{tabular}{|c|c|c|c|c|c|c|}
\hline & Apr 2017 & Jun 2017 & Aug 2017 & Sep 2017 & Nov 2017 & Feb 2018 \\
\hline TSH (mU/L) & $<0.02$ & $<0.02$ & $<0.02$ & $<0.02$ & $<0.02$ & $<0.02$ \\
\hline fT4 (pmol/L) & 49.7 & 29.3 & 19.4 & 18.8 & 18.4 & 15.7 \\
\hline
\end{tabular}

secondary to Graves' disease with strongly positive TRAB. Primary hypothyroidism once diagnosed usually requires lifelong thyroid hormone replacement. Although similar cases have been described in literature, none has been reported after such a long period of almost 30 years.

The first similar case was described by Joplin and Fraser in 1959 (4) and was followed by several others in the later 60s and 70s (5, 6, 7). In 1990 Takasu et al. (3) described a case series of eight cases converting to Graves' disease following Hashimoto's disease, and it was observed that those cases could be divided into three groups: a group of transient Graves' disease following hypothyroidism, a group of persistent Graves' thyrotoxicosis following hypothyroidism and a group of persistent hypothyroidism despite positive thyroidstimulating immunoglobulins.

What causes this conversion is not well understood, but there are different theories postulated. In this case, it happened after an upper respiratory tract infection, and this could implicate an environmental trigger in a genetically susceptible individual as a possible mechanism. One early study (6), as well as Takasu et al. (3) believe that the alterations in thyroid state were related to the balance in the activity of stimulating and blocking antibodies and the response of thyroid gland to these antibodies. More recently, Moriarty et al. (8) described a case of conversion of autoimmune hypothyroidism to hyperthyroidism with thyroid eye disease. They believe that a variable behaviour of the TRAB with the TSH receptor is responsible for the conversion from hypothyroidism to hyperthyroidism and vice versa. Furgan et al. (2) who also described three cases of autoimmune hypothyroidism converting to hyperthyroidism, proposed two theories to explain this conversion: first is the presence of both blocking and stimulating antibodies causing a pull-push effect shifting either to hypothyroidism or hyperthyroidism respectively, and a second theory is that thyroid damage from an autoimmune phenomenon initially causes thyroid hypofunction but once enough tissue has recovered, it is stimulated by stimulating antibodies. They also suggested that this conversion from hypothyroidism to hyperthyroidism may not be as rare as previously thought. One of the largest recent studies was done by Takasu and Matsushita (9). They identified 34 patients with hypothyroidism with TSH receptorblocking antibodies and 98 patients with Graves' disease with TSH receptor-stimulating antibodies and followed the natural course over 10 years. They concluded that hypothyroidism due to blocking antibodies and hyperthyroidism with stimulating antibodies may be two aspects of the same condition or disease.

We believe that genetically susceptible individuals may require an external trigger to switch from autoimmune hypothyroidism to Graves' disease, such as an infection or neck irradiation (10), which would cause the balance to shift from TSH receptor-blocking antibodies to TSH receptor-stimulating antibodies. This phenomenon may not be as rare as previously thought and the physicians should be aware of this possibility if someone with a previously well-established hypothyroidism is now requiring less or no thyroid hormone replacement, and the TFTs keep demonstrating an overactive picture with no obvious reason, even many years after the diagnosis of hypothyroidism as in this case. Further research, however, is needed to establish the exact pathogenesis of this phenomenon.

Patient's perspective

Patient's perspective available on demand.

\section{Declaration of interest}

The authors declare that there is no conflict of interest that could be perceived as prejudicing the impartiality of this case report.

\section{Funding}

This research did not receive any specific grant from any funding agency in the public, commercial or not-for-profit sector.

\section{Patient consent}

Written informed consent has been obtained from the patient for publication of the submitted article and accompanying images. 


\section{Author contribution statement}

Dr Kashif Hafeez was the lead clinician managing the patient's thyroid disorder; Dr Ehtasham Ahmad was the clinician trainee who wrote the initial main draft including discussion; Dr Apostolos Vrettos and Dr Muhammad Fahad Arshad went through the draft of the case report and suggested changes as need. Dr Apostolos Vrettos also drew the Figs 1 and 2.

\section{Acknowledgements}

The authors are especially thankful to our endocrine nurse Jimboy Isuga who made sure that all investigations were carried out as requested on time and he updated us accordingly.

\section{References}

1 Vanderpump MPJ. The epidemiology of thyroid disease. British Medical Bulletin 201199 39-51. (https://doi.org/10.1093/bmb/ldr030)

2 Furgan S, Haque NU \& Islam N. Conversion of autoimmune hypothyroidism to hyperthyroidism. BMC Research Notes 20147489. (https://doi.org/10.1186/1756-0500-7-489)

3 Takasu N, Yamada T, Sato A, Nakagawa M, Komiya I, Nagasawa Y \& Asawa T. Graves' disease following hypothyroidism due to Hashimoto's disease: studies of eight cases. Clinical Endocrinology 199033 687-698. (https://doi.org/10.1111/j.1365-2265.1990. tb03906.x
4 Joplin GF \& Fraser R. Thyrotoxicosis developing in recurrent nodular goitre with focal thyroiditis. Proceedings of the Royal Society of Medicine 195952 177-178.

5 Doniach D, Hudson RV \& Roitt LM. Human autoimmune thyroiditis: clinical studies. BMJ 19601 365-373. (https://doi.org/10.1136/ bmj.1.5170.365)

6 Goolden AWG, Davidson M \& Hoffenberg R. Myxedema preceding hyperthyroidism. Lancet 19712 268. (https://doi.org/10.1016/S01406736(71)92611-0)

7 Irvine WJ, Lamberg BA, Cullen D \& Raud-Gordin R. Primary hypothyroidism preceding thyrotoxicosis. Journal of Clinical and Laboratory Immunology 19798 3-19.

8 Moriarty M, Mills E, Yap HL \& Hamda A. Conversion of autoimmune hypothyroidism to hyperthyroidism with thyroid eye disease. Endocrine Abstracts 201537 EP1307. (https://doi.org/10.1530/ endoabs.37.EP1307)

9 Takasu N \& Matsushita M. Changes of TSH-stimulation blocking antibody (TSBAb) and thyroid stimulating antibody (TSAb) over 10 years in 34 TSBAb-positive patients with hypothyroidism and in 98 TSAb-positive Graves' patients with hyperthyroidism: reevaluation of TSBAb and TSAb in TSH-receptor-antibody (TRAb)-positive patients. Journal of Thyroid Research 20122012 182176. (https://doi. org/10.1155/2012/182176)

10 Lewandowski K, Dąbrowska K, Makarewicz J, Lewiński A. Pendulum swings from hypo- to hyperthyroidism: thyrotoxicosis after severe hypothyroidism following neck irradiation in a patient with a history of Hodgkin's lymphoma. Thyroid Research 20169 1. (https:// doi.org/10.1186/s13044-016-0030-1)

Received in final form 17 June 2018

Accepted 16 July 2018 